\title{
Modelling X-ray and Gamma-ray Emission from Pulsars
}

\author{
B. Rudak and J. Dyks \\ Nicolaus Copernicus Astronomical Center, Rabiańska 8, 87-100 Toruń, \\ Poland
}

\begin{abstract}
Lightcurves and broadband energy spectra of the brightest $\mathrm{X} / \gamma$-ray sources among the rotation powered pulsars exhibit unexpected richness of features, making each object almost a unique case. This contribution presents how our models of high-energy radiation within the framework of SCLF (space charge limited flow) polar-cap scenarios tackle with some of these challenges.
\end{abstract}

\section{Introduction}

Out of more than 1500 rotation powered pulsars known to date about forty were found to be the X-ray sources, and no more than and ten of them were detected in gamma-rays: the CGRO provided seven high-confidence detections with three other cases classified as 'likely' detections (e.g. Kanbach 2002); the gamma-ray sources were identified by virtue of flux pulsations with previously known $P$ and $\dot{P}$. No trace of pulsed signal in the VHE range $(300 \mathrm{GeV}-30 \mathrm{TeV})$ has been found so far for the gamma-ray pulsars. The high-energy lightcurves differ from those in radio. Their most striking feature are relatively long duty cycles as well as phase shifts in comparison to the radio pulses. Only for the Crab pulsar the peaks in gamma-rays and in radio overlap. The light-curve shapes fall into two categories. The Crab pulsar, Vela and Geminga show two sharp peaks separated in phase by $0.4-0.5$ and connected by an interpeak bridge of considerable level. B1706-44 shows two peaks separated by 0.2 in phase, with some hints of a third component in between. Other pulsars exhibit broad single pulses. The HE-radiation properties of pulsars challenge all theoretical models of pulsar magnetospheres and stimulate their development (see Baring (2001); Rudak, Dyks, \& Bulik (2002) for recent reviews). In this contribution we address briefly three recently elaborated aspects in modeling of HE-radiation properties: inverse Compton scattering of electron-positron pairs, rotational effects, and viewing geometry effects.

\section{Lightcurves and Broadband Spectra in the SCLF Models}

Magnetic inverse Compton scattering (mICS) of blackbody photons originating at the neutron star surface turned out to be a very promising way to tap part of kinetic energy from relativistic $\mathrm{e}^{ \pm}$pairs created via one-photon magnetic absorption. With energies between 10 and $1000 \mathrm{MeV}$ per particle, the pairs may contribute then considerably to the X-ray band of the pulsar energy spectrum. 

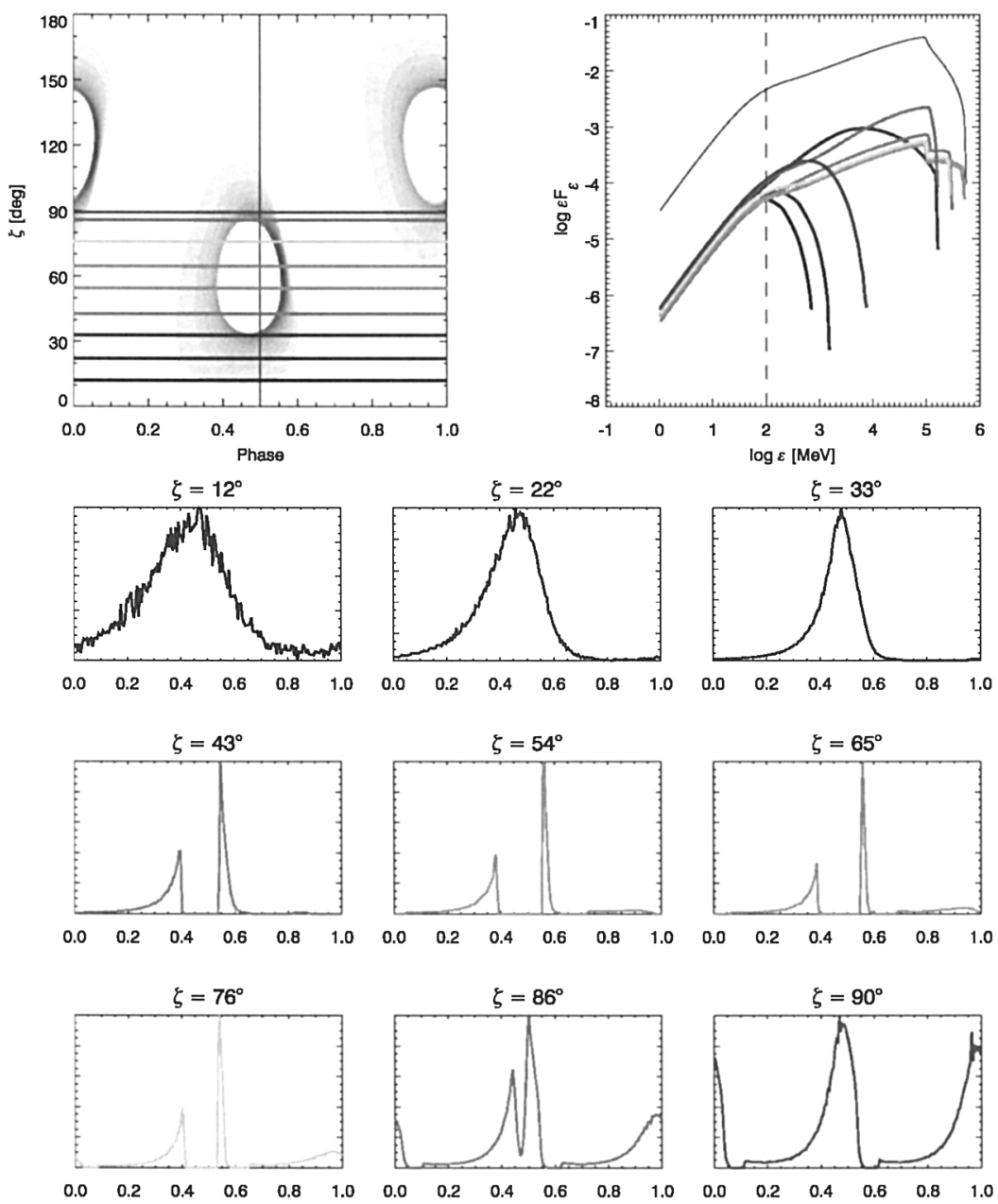

Figure 1. The characteristics of high energy radiation expected from a millisecond pulsar with $P=2.3 \mathrm{~ms}, B=10^{9} \mathrm{G}$ and the magnetic axis inclination $\alpha=60^{\circ}$. The top left panel shows the intensity distribution for radiation above $100 \mathrm{MeV}$ as a function of rotational phase $\phi$ and viewing angle $\zeta$. Horizontal color-coded lines correspond to the position of nine observers located at different angles $\zeta$. For each $\zeta$ the phase-averaged spectrum drawn with color-coded line is shown in top right panel. The corresponding pulse profiles are shown in small panels labelled with $\zeta$. The spectrum of total luminosity from the pulsar (thin solid line in top right panel) is plotted (at an arbitrary level relative to the phase-averaged spectra) for comparison of the shapes. (From Woźna et al. 2002). 


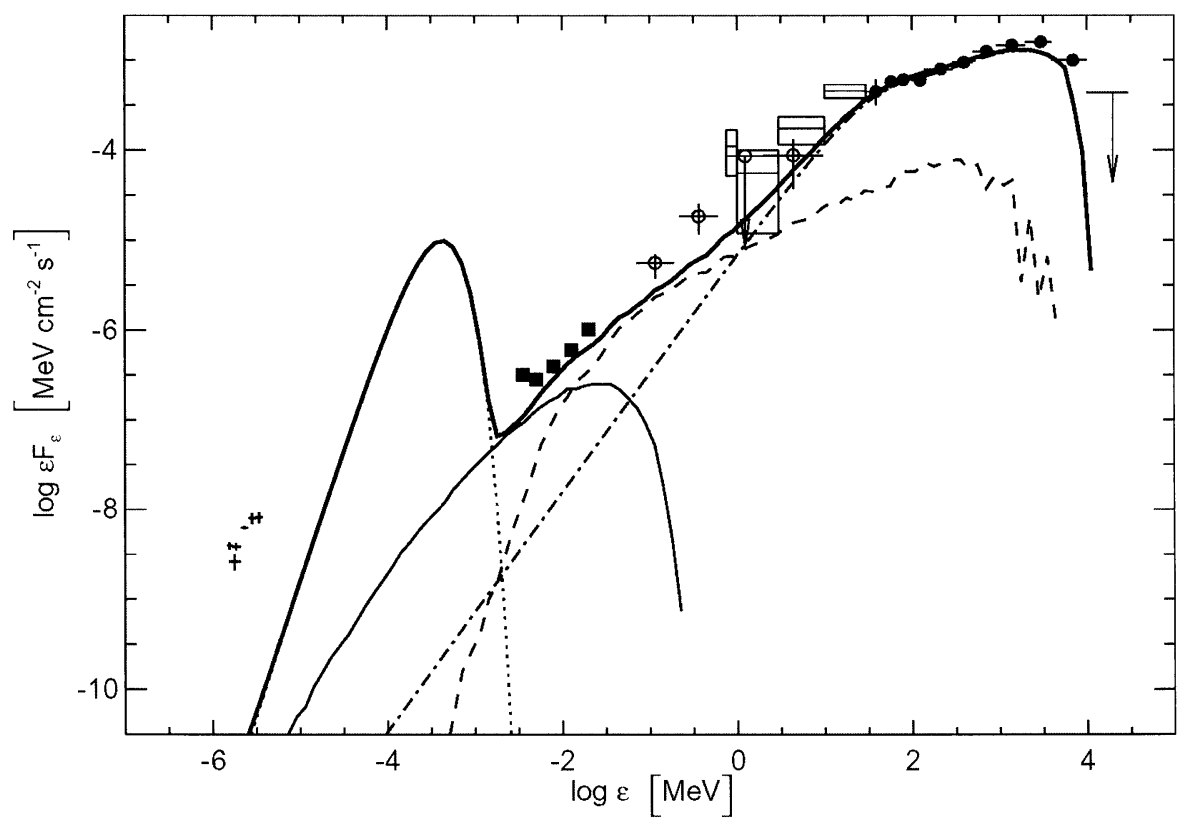

Figure 2. The model energy spectrum calculated to reproduce spectral features of the Vela pulsar $\left(P=89 \mathrm{~ms}, B_{\mathrm{s}}=6 \times 10^{12} \mathrm{G}\right)$. The broad-band spectrum consists of four components due to: curvature radiation of primary electrons (dot-dashed), synchrotron radiation of secondary $e^{ \pm}$-pairs (dashed), inverse Compton scattering of surface Xray photons on the $e^{ \pm}$-pairs (thin solid) and the blackbody surface emission (dotted). The surface temperature $T_{\mathrm{s}}=1.26 \times 10^{6} \mathrm{~K}$ was assumed for the neutron star. Total spectrum is given by a thick solid line. Phase-averaged data points for Vela from different telescopes are indicated (for details see Figs. 4 and 5 of Rudak et al. (2002)).

It was Zhang \& Harding (2000) who recognized this particular scenario and offered an approximate analytical estimates for the resulting X-ray luminosity. A necessary extension of this approach was done with numerical Monte Carlo simulations of mICS for the $\mathrm{e}^{ \pm}$pairs (Dyks, Rudak, \& Bulik 2001). The numerical model was applied to the Vela pulsar. Our Monte Carlo approach presents clearly in Fig.2 an important role of the mICS component in the overall energy spectrum; without this component it would be difficult to reproduce the RXTE data points for the Vela pulsar (filled squares in Fig.2).

Pulsar rotation is able to affect the double-peak gamma-ray lightcurves above $5 \mathrm{GeV}$ under favorable conditions. Dyks \& Rydak (2002) showed that pulsar rotation induces asymmetry in the one-photon magnetic absorption rate with respect to the magnetic dipole axis. The asymmetry between the leading peak and the trailing peak developes via asymmetric increase with altitude of rotationinduced electric field with respect to local magnetic field, as well as slippage of magnetic field lines under the photon's path. The resulting features - the softer 
spectrum of the leading peak and the dominance of the trailing peak above $\sim 5 \mathrm{GeV}$ - agree qualitatively with what has been noted recently for the bright gamma-ray pulsars (Thompson 2001).

Viewing geometry has been recognized as an important factor in shaping the lightcurves as well as broadband energy spectra of pulsars as well as playing potentially important role in the context of EGRET UID galactic sources (Baring 2001, Harding \& Zhang 2001). A variety of apparent pulsar characteristics is possible upon changing the viewing angle $\zeta$. Fig.1 presents a case of a millisecond pulsar with the effects of orientation and rotation combined (after Woźna et al. 2002). The lightcurves are energy-integrated above $100 \mathrm{MeV}$ and the gamma-ray spectra are two-component: due to curvature and synchrotron emission; inverse Compton scattering was neglected. The effects of aberration and light travel delays are important for millisecond pulsars, and affect their lightcurves, especially at large inclination angles $\alpha$. Whenever the line of sight misses outer rim of the polar cap, the calculated spectra become soft with an apparent exponential (and not super-exponential) high-energy cutoff, mimicking thus an outer-gap candidate. The millisecond gamma-ray pulsar J0218+4232 (Kuiper et al. 2002) is probably a good candidate for such a case.

Acknowledgments. BR acknowledges financial support from KBN (grant 2P03D02117) and IAU.

\section{References}

Baring, M. G. 2001, Proc. Tonantzintla Workshop, eds. A. Carramiñana, O. Reimer, D.J. Thompson, Ap\&SS Library, 267, 167

Dyks, J. \& Rudak, B. 2002, Proc. of the XXII Moriond Meeting, in press (astro-ph/0205222)

Dyks, J. \& Rudak, B. 2002, A\&A, 393, 511

Dyks, J., Rudak, B., \& Bulik, T. 2001, Proc. 4th INTEGRAL Workshop, ed. A. Gimenez, V. Reglero, \& C. Winkler, ESA SP-459, 191

Harding, A. K. \& Zhang, B. 2001, ApJ, 548, L37

Kanbach, G. 2002, Proc. 270. WE-Heraeus Seminar on Neutron Stars, Pulsars and Supernova Remnants. MPE Report 278. eds. W. Becker, H. Lesch, J. Truemper, p.91

Kuiper, L., Hermsen, W., Verbunt, F., et al. 2002, ApJ, 577, 917

Rudak, B., Dyks, J., \& Bulik, T. 2002, Proc. 270. WE-Heraeus Seminar on Neutron Stars, Pulsars and Supernova Remnants. MPE Report 278. eds. W. Becker, H. Lesch, J. Truemper, p.142

Thompson, D. J. 2001, Gamma-Ray Pulsars: Observations. In International Symposium on High Energy Gamma-Ray Astronomy, ed. F. A. Aharonian, \& H. J. Völk, AIP Conference Proceedings, vol. 558, 103

Woźna, A., Dyks, J., Rudak, B., \& Bulik, T. 2002, Proc. of the XXII Moriond Meeting, in press (astro-ph/0205224)

Zhang, B. \& Harding, A. K. 2001, ApJ, 532, 1150 